\title{
Rapid pedobarographic image registration based on contour curvature and optimization
}

\author{
Francisco P. M. Oliveira \\ Faculdade de Engenharia da Universidade do Porto \\ Rua Dr. Roberto Frias \\ 4200-465 Porto \\ Portugal \\ francisco.oliveira@fe.up.pt \\ João Manuel R. S. Tavares \\ Departamento de Engenharia Mecânica \\ Faculdade de Engenharia da Universidade do Porto \\ Rua Dr. Roberto Frias \\ 4200-465 Porto \\ Portugal \\ tavares@fe.up.pt \\ Todd C. Pataky \\ HACB, School of Biomedical Sciences \\ University of Liverpool \\ Sherrington Buildings, Ashton Street \\ Liverpool, L69 3GE \\ UK \\ tpataky@liv.ac.uk
}

\section{Corresponding author:}

Professor João Manuel R. S. Tavares

Departamento de Engenharia Mecânica

Faculdade de Engenharia da Universidade do Porto

Rua Dr. Roberto Frias

4200-465 Porto

Portugal

Phone: +351 225081487

Fax: +351225081445

Email: tavares@fe.up.pt 


\section{Rapid pedobarographic image registration based on contour curvature and optimization}

Abstract: Image registration, the process of optimally aligning homologous structures in multiple images, has recently been demonstrated to support automated pixel-level analysis of pedobarographic images and, subsequently, to extract unique and biomechanically relevant information from plantar pressure data. Recent registration methods have focused on robustness, with slow but globally powerful algorithms. In this paper, we present an alternative registration approach that affords both speed and accuracy, with the goal of making pedobarographic image registration more practical for near-real-time laboratory and clinical applications. The current algorithm first extracts centroid-based curvature trajectories from pressure image contours, and then optimally matches these curvature profiles using optimization based on dynamic programming. Special cases of disconnected images (that occur in high-arched subjects, for example) are dealt with by introducing an artificial spatially linear bridge between adjacent image clusters. Two registration algorithms were developed: a 'geometric' algorithm, which exclusively matched geometry, and a 'hybrid' algorithm, which performed subsequent pseudo-optimization. After testing the two algorithms on 30 control image pairs considered in a previous study, we found that, when compared with previously published results, the hybrid algorithm improved overlap ratio ( $p=0.010$ ), but both current algorithms had slightly higher mean-squared error, assumedly because they did not consider pixel intensity. Nonetheless, both algorithms greatly improved the computational efficiency ( $25 \pm 8 \mathrm{~ms}$ and $53 \pm 9$ ms per image pair for geometric and hybrid registrations, respectively). These results imply that registration-based pixel- 
level pressure image analyses can, eventually, be implemented for practical clinical purposes. 


\section{Introduction}

Recent pedobarographic studies have demonstrated that pixel-level approaches can, in some situations, more effectively extract biomechanically-relevant information from plantar pressure images than traditional regional techniques (Pataky et al., 2008a). Since the foot adopts an arbitrary posture with respect to pedobarographic equipment, image registration, the process of optimally aligning images, is essential to such pixel-level analyses. Registration of plantar pressure images has been conducted previously (Harrison and Hillard, 2000; Tavares et al., 2000; Pinho and Tavares, 2004; Pataky et al., 2008b), but these studies mainly examined the scientific plausibility of registrationbased analyses and did not focus specifically on implementing registration in a way that would be practical in both the laboratory and clinic.

The goal of the current paper was thus to develop a pedobarographic registration algorithm that, because of negligible execution time and robustness to natural pressure image variation, could afford practical adoption in routine plantar pressure measurement sessions. .To this end, we currently modify and improve previous algorithms (Oliveira and Tavares, 2008; Oliveira and Tavares, 2009) for speed and accuracy.

\section{Methods}

\subsection{Dataset}

Data from a previous study (Pataky et al., 2008b) were re-analyzed here to afford direct comparison of the current and previous algorithms. The dataset consisted of 30 pairs of peak pressure images, three random image pairs from 10 random subjects; only 30 image pairs were analyzed because manual registration was also tested in the cited study. The data were originally collected at $500 \mathrm{~Hz}$ using a $0.5 \mathrm{~m}$ Footscan system (RSscan, Olen, Belgium) 


\subsection{Contour-based geometric registration}

The algorithm consisted of four steps (Fig.1): (1) extract image contours, (2) assemble contour affinity matrix, (3) optimize contour points matching, and (4) compute transformation parameters. Image contours, piecewise-continuous collections of $(x, y)$ coordinates that represent constant pressure, were extracted in four sub-steps: binarize (with a pressure threshold $\delta>0$ ); spatially smooth (using morphological closing with a circular mask of radius 3) (Haralick and Shaprio, 1992); extract boundary points; define subcontours (Fig. 2.b); build global virtual contours (Fig. 2.c).

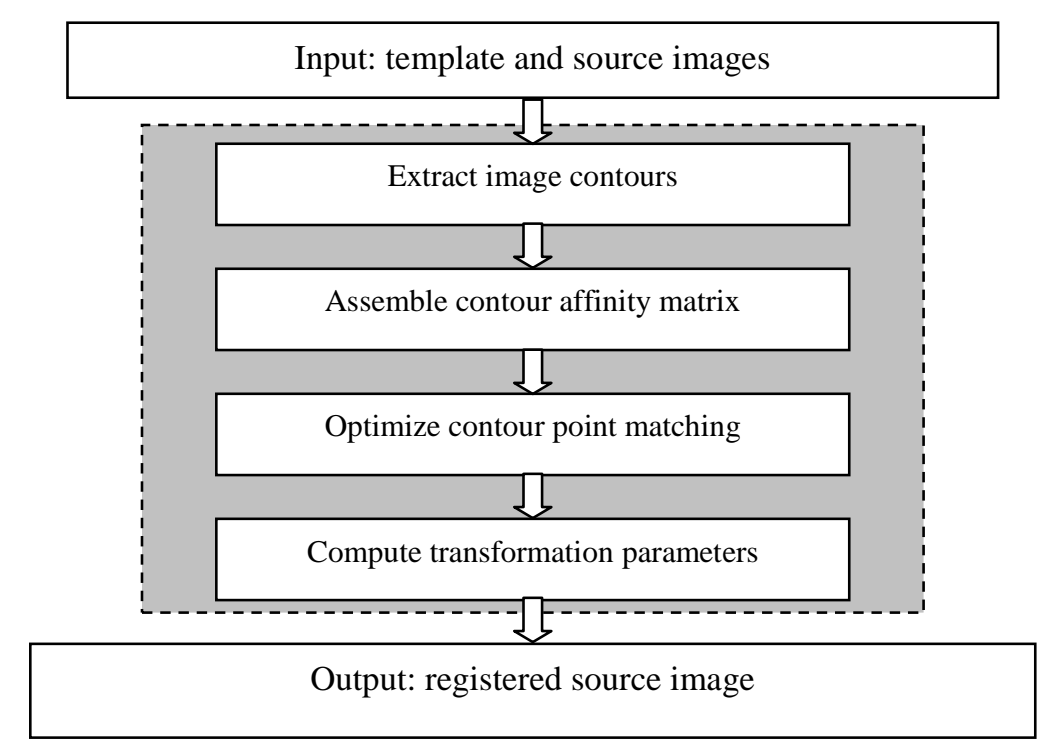

Figure 1: Registration algorithm schematic.

Next, following Oliveira and Tavares (2009), we assembled a contour affinity matrix $C$ which describes the similarity between template and source contours based on each point curvature value and its distance to the associated centroid. Each element of $C$ is given by:

$c_{i j}=w \times\left\|d t_{i}, d s_{j}\right\|+(w-1) \times\left|\alpha_{i}-\theta_{j}\right| \times \bar{X}$, 
where $w$ represents a distance-to-centroid weighting parameter (in this case, 0.5 ), $d t_{i}$ and $d s_{j}$ represent the normalized distances of point $i$ and $j$ of template and source contours, respectively, to the contour centroid; $\left|\alpha_{i}-\theta_{j}\right|$ represents the curvature difference at points $i$ of the template contour $\left(\alpha_{i}\right)$ and $j$ of the source contour $\left(\theta_{j}\right)$, and $\bar{X}$ represents the average of all curvature values. Thus, each $c_{i j}$ represents the matching cost between point $i$ of the template contour and point $j$ of the source contour, where high values indicate low affinity between the respective points.

Third, we used dynamic programming (Oliveira and Tavares, 2008) to minimize global affinity and thus find optimal contour matching from $C$ (Fig. 2). Finally, we estimated the three optimal transformation parameters (two translational, one rotational) according to Oliveira and Tavares (2009).

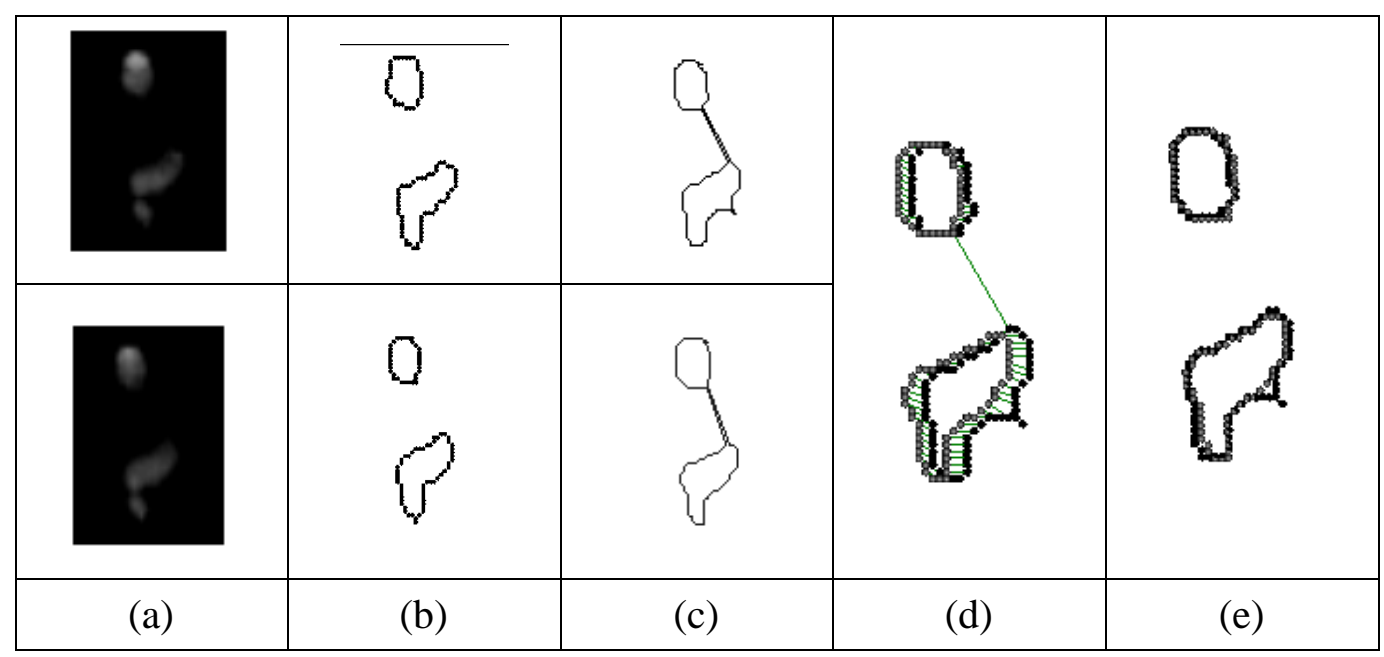

Figure 2: Global matching between two contours of a foot: (a) original images; (b) subcontours (without links among points); (c) virtual global contours; (d) contours in original position; (e) registered contours. (The dots represent the contours' pixels and the thin lines indicate the established correspondences). 


\subsection{Hybrid registration}

A hybrid algorithm used the geometric solution as an initial state for subsequent threeparameter pseudo-optimization. Here the geometrically registered source image was transformed by all 27 combinations of rotation $\left(-0.25^{\circ}, 0^{\circ},+25^{\circ}\right)$ and $x, y$ translations $(-$ $0.5,0,0.5$ pixels). The $x, y$, rotation combination that minimized the exclusive-or (XOR) and mean-squared-error (MSE) dissimilarity metrics (Pataky et al., 2008b) were both selected for subsequent analysis. The current algorithms were implemented in C++, using Microsoft Visual Studio 6 and were tested on a notebook PC (AMD Turion64 2.0GHz, 1.0GB, Microsoft Windows XP).

\subsection{Registration accuracy assessment using control images}

We assessed registration accuracy by applying arbitrary known transformations to the set of 30 template images and then comparing these known parameters to the ones obtained using the geometric algorithm (Section 2.2). We did not test the hybrid algorithm (Section 2.3) in this manner, because the intention of this accuracy assessment was only to emphasize the quality of the initial contour-based registration.

\subsection{Registration quality assessment using experimental images}

Since the main goal was to develop a fast and accurate algorithm, both speed (ms) and accuracy (MSE, XOR) were computed for all experimental image pairs. Speed was compared qualitatively and accuracy, statistically using two-sided $t$ tests, with the global MSE and XOR algorithms of Pataky et al. (2008b). The sensitivity of XOR and MSE was assessed qualitatively for several threshold levels $\delta$. 


\section{Results}

\subsection{Speed}

Average registration durations (ms) per image pair were: geometric: $25 \pm 8$; hybrid: $53 \pm 9$. These durations include all tasks, from disk data reading to final image building (Fig. 1).

\subsection{Accuracy}

The current geometric algorithm was highly accurate for known transformations (Table $1)$.

Table 1: Registration accuracy for controlled (known) transformations. Only results for threshold level $\delta=0.001 \mathrm{~N} / \mathrm{cm}^{2}$ are presented because significant differences were not observed for threshold values $0.001 \leq \delta \leq 1 \mathrm{~N} / \mathrm{cm}^{2}$.

\begin{tabular}{|c|c|c|c|c|c|}
\hline \multicolumn{2}{|c|}{ Transformation control } & \multicolumn{4}{|c|}{$\begin{array}{c}\text { Transformation estimate } \\
\left.\text { (using } \delta=0.001 \mathrm{~N} / \mathrm{cm}^{2}\right)\end{array}$} \\
\hline $\begin{array}{c}\text { Angle } \\
{\left[{ }^{\circ}\right]}\end{array}$ & $\begin{array}{c}\text { Translation } \\
\text { [pixel] }\end{array}$ & $\begin{array}{c}\text { Angle } \\
{\left[{ }^{\circ}\right]}\end{array}$ & $\begin{array}{c}\text { Translation } \\
{[\text { pixel] }}\end{array}$ & $\begin{array}{c}\text { XOR } \\
{[\%]}\end{array}$ & $\begin{array}{c}\text { MSE } \\
{\left[\left(\mathrm{N} / \mathrm{cm}^{2}\right)^{2}\right]}\end{array}$ \\
\hline 0 & $(-7,-2)$ & 0 & $(-7,-2)$ & $0 \pm 0$ & $0 \pm 0$ \\
\hline-8 & $(0,0)$ & $-7.8 \pm 0.5$ & $(0.1 \pm 0.3,-0.1 \pm 0.3)$ & $19.0 \pm 1.5$ & $1.6 \pm 1.7$ \\
\hline-20 & $(-5,8)$ & $-19,9 \pm 0.5$ & $(-4.9 \pm 0.3,8.0 \pm 0.3$ & $19.5 \pm 1.6$ & $1.5 \pm 1.2$ \\
\hline
\end{tabular}

The geometric and hybrid algorithms also produced good-quality registration for unknown experimental transformations (Figs. 3 and 4), when compared with the (slow) global search methods used in previous study. We found that, although the geometric algorithm did not significantly improve XOR ( $p=0.218)$ with respect to the previous study, the hybrid algorithm did $(p=0.010)$. Both algorithms produced lower MSE $(\delta=1.0, p<0.001)$. 

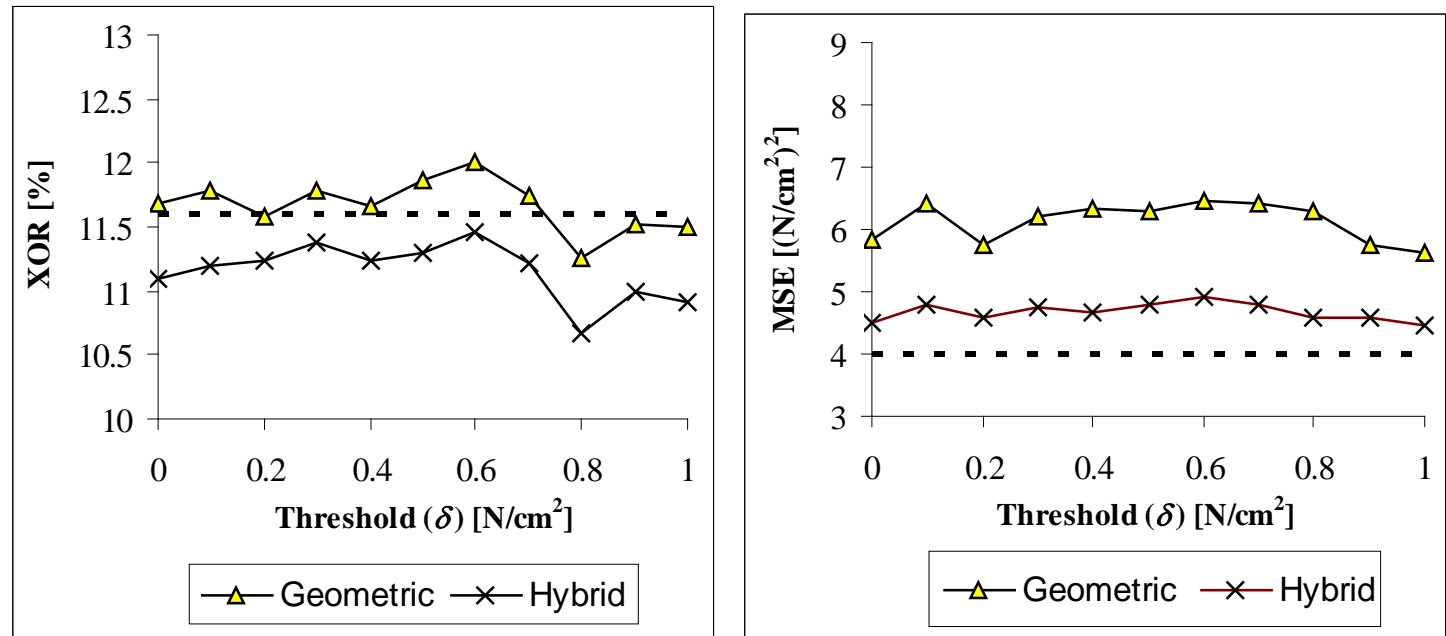

Figure 3: Registration accuracy. (Dotted horizontal lines represent the best value obtained by Pataky et al. (2008b).)
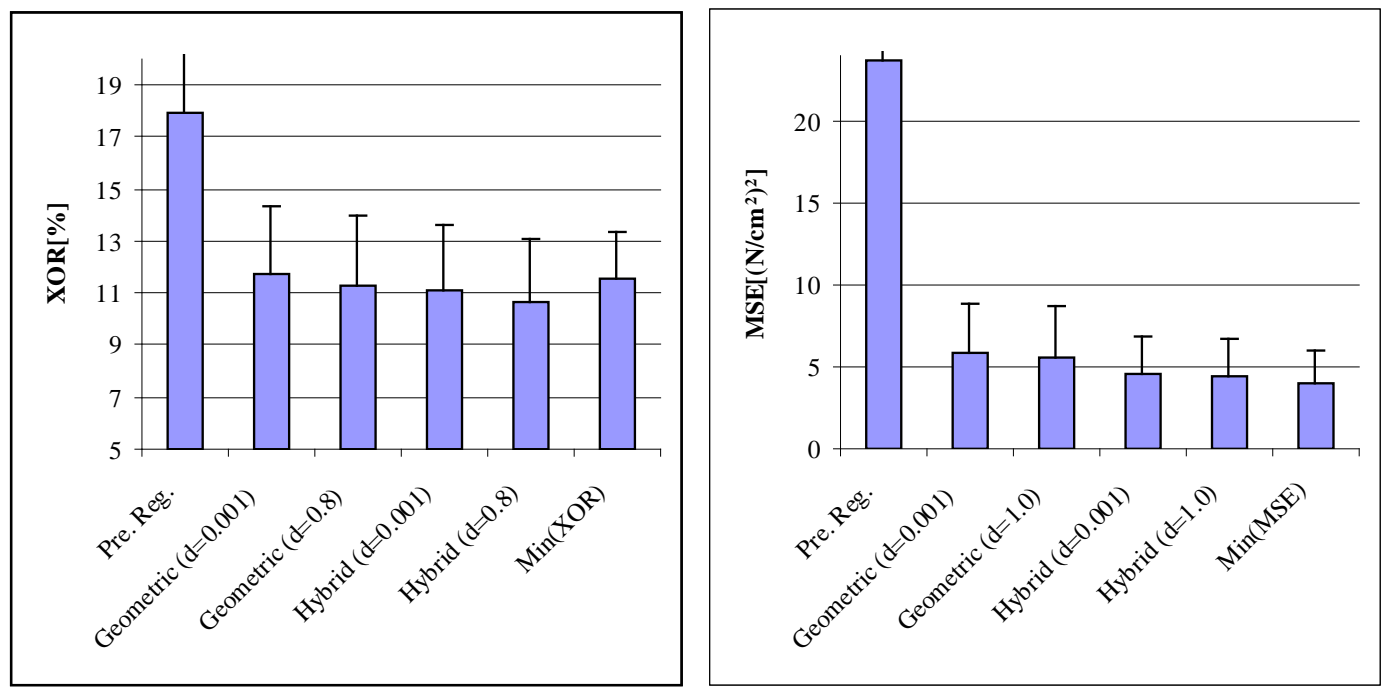

Figure 4: Registration accuracy comparison: geometric, hybrid, and Pataky et al. (2008b); ‘Min(XOR)’ and 'Min(MSE)’ refer to the results of Pataky et al. (2008b).

\section{Discussion}

The current results demonstrate that both the geometric and hybrid registrations were very rapid and were also robust to natural variation in a small random sample of nonpathologic pedobarographic images. The geometric algorithm was accurate for known transformations (Table 1); the small observed errors were expected because geometric 
image transformations produce subtle nonlinear distortions due to interpolation imperfections (e.g. Parker et al., 1983). Indeed, the geometric algorithm tended to produce better XOR values than Pataky et al. (2008b). Although the cited study employed powerful global optimization, the current results can be partially attributed the fact that Pataky et al. (2008b) used smoothed images, implying that the computed transformation parameters were not optimal for the original (unsmoothed) images. For the current hybrid algorithm, the XOR results were better than the best result presented in Pataky et al. (2008b) for all values of $\delta$ tested. Both current algorithms produced slightly higher MSE values than Pataky et al. (2008b), but this was expected because the current algorithms are shape-based, not pixel-intensity-based. The main point here is that the current algorithm performed very similar to a global algorithm, but did so very quickly, at speeds rapid enough for practical clinical use.

We currently considered only threshold levels $\delta$ smaller than $1.0 \mathrm{~N} / \mathrm{cm}^{2}$ because we found that higher thresholds yielded contours that became decreasingly representative of characteristic foot shape and, consequently, were not suitable for shape-based image alignment. For the range of current thresholds, the ultimate alignments were not appreciably different (Fig. 3), demonstrating that this parameter does not have a significant effect on the alignment quality.

Frequently, because we forced the matching of all contour points defined by smaller number of points, one or two correspondences found were wrong (Fig. 2.d). We did not eliminate those correspondences before computing the rigid transformation. The reason is that we used the median, a parameter robust to outliers, to estimate the global geometric transformation.

The computational time required by the geometric algorithm was very low, in the order of $25 \mathrm{~ms}$, which suggests that it may be valuable for clinical applications, where data 
processing time is limited. For example, automated registration-based analyses such as those employed herein may be used by the clinician to directly compare a given image with previous images and/or with other individuals' images. When seeking speed for practicality, however, one must be aware that accuracy may suffer. This speed-accuracy trade-off is demonstrated by the current hybrid algorithm, which performed more accurately but more slowly than the purely geometric algorithm. Nonetheless, accuracy performances that are comparable to those of much slower previous algorithms (Fig. 4) suggest that the current algorithm is the fastest and most accurate algorithm proposed to date. Indeed, its speed and accuracy both appear to be adequate for practical clinical use.

The current registration procedure relies on boundary points to define image contours. This approach followed previous registration successes, (e.g. Shapiro and Brady, 1992; Sclaroff and Pentland, 1995; Bastos and Tavares, 2006; Scott and Nowak, 2006; Gonçalves et al., 2008; Oliveira and Tavares, 2008; Vasconcelos and Tavares, 2008; Oliveira and Tavares, 2009) that were mainly based on contour extraction. In future work we intend to test additional algorithms that do not force over-matching and that involve alternative hybrid registration methodologies. Because these optimizations will be based only on local search, we expect that they will continue to be faster than the previous global search-based methods and thus maintain their potential clinical utility. In summary, contour-based matching provides a very rapid initial registration which, when augmented by local optimization, performs at speeds and accuracies that appear to be adequate for routine plantar pressure measurement sessions. Practicality, utility, and robustness to arbitrary pedobarographic variation, especially in clinical populations, will be assessed in future studies with working-clinic implementation trials. 


\section{Acknowledgements}

The first author would like to thank his PhD grant of the Portuguese Fundação Calouste Gulbenkian. The third author wishes to acknowledge financial support from the Leverhulme Trust (Grant F/0025/x) and NERC (Grants GR3/11202 and GR3/12004).

\section{References}

Bastos, L., Tavares, J. M. R. S., 2006. Matching of objects nodal points improvement using optimization. Inverse Problems in Science and Engineering 14(5), 529-541.

Gonçalves, P., Tavares, J. M. R. S., Jorge, R. N., 2008. Segmentation and simulation of objects represented in images using physical principles. Computer Modeling in Engineering \& Sciences 32(1), 45-55.

Haralick, R.M., Shapiro, L.G., 1992. Computer and Robot Vision. Addison-Wesley, Reading.

Harrison, A. J., Hillard, P. J., 2000. A moment-based technique for the automatic spatial alignment of plantar pressure data. Proceedings of the Institute of Mechanical Engineers, Part H: Journal of Engineering in Medicine 214(3), 257-264.

Oliveira, F., Tavares, J. M. R. S., 2008. Algorithm of dynamic programming for optimization of the global matching between two contours defined by ordered points. CMES: Computer Modeling in Engineering \& Sciences 31(1), 1-11.

Oliveira, F., Tavares, J. M. R. S., 2009. Matching contours in images through the use of curvature, distance to centroid and global optimization with order-preserving constraint. CMES: Computer Modeling in Engineering \& Sciences 43(1), 91-110.

Parker, J., Kenyon, R., Troxel, D., 1983. Comparison of interpolating methods for image resampling. IEEE Transactions on Medical Imaging MI-2(1), 31-39. 
Pataky, T. C., Caravaggi, P., Savage, R., Parker, D., Goulermas, J. Y., Sellers, W. I., Crompton, R. H., 2008a. New insights into the plantar pressure correlates of walking speed using pedobarographic statistical parametric mapping. Journal of Biomechanics 41(9), 1987-1994.

Pataky, T. C., Goulermas, J., Crompton, R., 2008b. A comparison of seven methods of within-subjects rigid-body pedobarographic image registration. Journal of Biomechanics 41(14), 3085-3089.

Pinho, R., Tavares J. M. R. S., 2004. Dynamic pedobarography transitional objects by Lagrange's equation with FEM, modal matching and optimization techniques. Lecture Notes in Computer Science 3212, Image Analysis and Recognition. Springer, Berlin, 92-99.

Sclaroff, S., Pentland, A., 1995. Modal matching for correspondence and recognition. IEEE Transactions on Pattern Analysis and Machine Intelligence 17(6), 545-561.

Scott, C., Nowak, R., 2006. Robust contour matching via the order-preserving assignment problem. IEEE Transactions on Image Processing 15(7), 1831-1838.

Shapiro, L., Brady, M., 1992. Feature-based correspondence: an eigenvector approach. Image and Vision Computing 10(5), 283-288.

Tavares, J. M. R. S., Barbosa, J., Padilha, A. J., 2000. Matching image objects in dynamic pedobarography. In Proceedings of the 11th Portuguese Conference on Pattern Recognition. Porto, Portugal.

Vasconcelos, M., Tavares, J. M. R. S., 2008. Methods to automatically built point distribution models for objects like hand palms and faces represented in images. Computer Modeling in Engineering \& Sciences 36(3), 213-241. 